\title{
Screening of targeted genes in extrahepatic bile ducts of mice with experimental biliary atresia
}

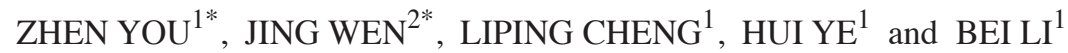 \\ ${ }^{1}$ Department of Biliary Surgery, West China Hospital, Chengdu, Sichuan 610041; \\ ${ }^{2}$ Campus Hospital, Sichuan University, Chengdu, Sichuan 610000, P.R. China
}

Received May 7, 2014; Accepted February 13, 2015

DOI: $10.3892 / \mathrm{mmr} .2015 .3903$

\begin{abstract}
Biliary atresia (BA) is an infantile disease resulting from a severe cholangiopathy, which can obstruct extrahepatic bile ducts, disrupt bile flow and lead to end-stage cirrhosis. The current study aimed to develop a genetic method to investigate the pathogenesis of BA. The gene expression profile of BA (GSE46967) was downloaded from the Gene Expression Omnibus database and included 18 samples from newborn mice. These samples were collected at three time points following the induction of BA with rhesus rotavirus. The differentially expressed genes (DEGs) in mice with BA were identified using the limma package in $\mathrm{R}$ language, followed by hierarchical clustering analysis. Gene ontology functional analysis and Kyoto Enrichment of Genes and Genomes pathway analysis of the selected common DEGs was conducted using the Database for Annotation, Visualization and Integrated Discovery. In total, 306 DEGs were identified in the samples from the 3 day time point, 721 at 7 days and 370 at 14 days. A total of 74 common DEGs were identified in these three sample groups, which are reported to function in multiple immune biological processes, including the defense response, leukocyte migration, cell chemotaxis and leukocyte chemotaxis. In addition, 'cytokine-cytokine receptor interaction' and 'chemokine signaling pathway' were observed to be significantly enriched in BA. A total of six common DEGs (CCL3, CXCL5, CXCL13, CXCR2, CCL5 and CCL6) were identified that were involved in the significantly enriched functions and the significantly enriched pathways. The data from the current study suggested that the immune response is a critical biological process in the development of BA. The six critical hub genes identified (CCL3, CXCL5, CXCL13,
\end{abstract}

Correspondence to: Dr Hui Ye, Department of Biliary Surgery, West China Hospital, 37 Guo Xue Ally, Chengdu, Sichuan 610041, P.R. China

E-mail: huiyehy@163.com

*Contributed equally

Key words: biliary atresia, differentially expressed genes, function enrichment, pathway analysis
CXCR2, CCL5 and CCL6) may be used as specific target genes in the treatment of BA.

\section{Introduction}

Biliary atresia (BA) is a rare hepatobiliary disorder in infants with poor prognosis caused by an obstruction of the bile ducts, leading to a disruption in bile flow, which can rapidly progress to biliary cirrhosis $(1,2)$. The incidence of BA in Asia is higher than in western countries, with reports indicating a rate of $1 / 9,600$ to $1 / 16,700$ of live births (3). BA is reported to occur in half of all cases of neonatal cholestasis with a greater incidence in females, and associated congenital malformations occur in $3-10 \%$ of cases of BA (4). Without treatment, BA progresses into liver cirrhosis, hepatic failure and mortality in $<2$ years following birth $(5,6)$. Natural mortality of BA is $100 \%$, therefore liver transplantation is a necessary requirement for late stage BA (7); however, current medical management of patients with BA is unsatisfactory, placing an economic burden on society and family members $(5,7)$.

The etiology of BA remains to be fully elucidated; however, previous studies have identified three factors suggested to contribute to the formation of BA. One is congenital genetic abnormalities, for example, the mutation of INVS gene, which leads to abnormal functioning of inversin, and may terminate the development of biliary ducts during the embryonic period (8). Another factor identified by various studies is viral infection (9,10), with cytomegalovirus, Epstein-Barr virus and human papilloma virus having all been isolated from the livers of infants with BA (11-13). The third factor identified was immune disorders, which appeared following the viral infection (14). Previous studies have supported the hypothesis that the viral infection triggering host immune responses may be the basis for the pathogenesis of BA (15). Leonhardt et al (16) and Schreiber and Kleinman (17) additionally agreed that $\mathrm{BA}$ is a virus-induced, immune-mediated disease that occurs in genetically susceptible individuals. Previous studies have demonstrated that BA was associated with $\mathrm{T}$ helper cell response type I-mediated portal tract inflammation and that interleukin (IL)-2, interferon $\gamma$, IL-12, tumor necrosis factor $\alpha$ and macrophage activation all served important roles $(18,19)$. The current study aimed to elucidate the pathogenesis of BA using biological information analysis in relation to immunology. 
Microarray analysis has been reported as an effective method to monitor alterations in gene expression and identify genes that are associated with biological processes induced by BA. Leonhardt et al (16) identified several targeted genes that were implicated in the development of BA, including CCL2, CCL5, CCR5, CXCL9, CXCL10 and IL1F5 (20). The majority of these genes were associated with the immune system or with modulating inflammation.

Previous studies have demonstrated that the expression levels of cell signaling and transcription regulatory genes, such as CIT or ActRII were upregulated during BA progression (21), and BA was observed to be a necrotizing process caused by viral cholangitis at the embryonic stage and the expression level of the type II major histocompatibility complex was increased in the livers of patients (22). However, there is little evidence indicating the relevance between human leukocyte expression and BA. Thus, the cause and pathogenetic mechanism of BA remain largely unknown.

In the present study, microarray analysis was used to monitor differentially expressed genes (DEGs) in BA samples at different time points (3,7 and 14 days) following induction with rhesus rotavirus (RRV) compared with sham-RRV-control. Comprehensive bioinformatics analysis was used, which combined gene ontology (GO) and Kyoto Enrichment of Genes and Genome (KEGG) pathway information, in order to provide a more thorough understanding of the biological mechanisms of BA. The current study aimed to predict the hub genes that are most likely to be associated with BA, and to identify their molecular mechanisms in BA. This may aid in the development of novel therapeutic strategies for BA.

\section{Materials and methods}

Affymetrix microarray data. The microarray data were obtained from the Gene Expression Omnibus (GEO; http://www.ncbi. nlm.nih.gov/geo/) database from the National Center for Biotechnology Information (Bethesda, MD, USA) (23), which is the largest public database of its type. The gene expression profiles were extracted from the GEO database (ID: GSE46967) based on the GPL6246 Affymetrix Mouse Gene 1.0 ST array (Affymetrix, Inc., Santa Clara, CA, USA). The experimental mice (18 tissue samples) were injected intraperitoneally with $1.5 \times 10^{6}$ units of rhesus rotavirus (RRV) or $0.9 \%$ normal saline (NS; control) within $24 \mathrm{~h}$. The microarray data were collected from the experimental group and the control group at 3, 7 and 14 days after RRV or saline injection. Each experimental condition contained three sets of samples (three treated with RRV three with normal saline).

A total of 28,816 probes were mapped with the gene names labelled in the GPL6246 annotation platform, and a log 2 transformation was performed on 25,299 genes (24). The limma package (http://bioinf.wehi.edu.au/limma) in $\mathrm{R}$ language was used to select the DEGs of the case-group compared with the control-group (25). The P-values were adjusted by the Benjamin and Hochberg method, and the FDR-value based on the multtest package of 0.05 was used as the cut-off criterion (26).

Hiarchical clustering of DEGs. The expression values of DEGs in each group were selected according to the probe information from the downloaded file. The R language pheatmap package (http://cran.r-project.org/web/packages/pheatmap/index.

html) was used for hierarchical clustering analysis (27) of DEGs based on Euclidean distance (28), and the results were presented in heat maps. Genes with similar expression levels could be collected together by hierarchical clustering for a convenient search. Samples clustered based on the gene expression value can aid in determining whether the screening DEGs have sample specificity or not, which means the selected sample types (case or control) can be identified based on the differential gene expression information.

Common DEGs of three time points. The expression levels of selected genes from samples at 3, 7 and 14 days were compared, in order to identify the common DEGs (29).

GO analysis and pathway enrichment analysis. GO analysis has become a commonly used approach for functional studies of large-scale genomic or transcription data (30). The KEGG pathway database contains information of how molecules or genes are networked, which is complementary to the majority of the existing molecular biology databases containing the information of individual genes (31). The Database for Annotation, Visualization and Integrated Discovery (DAVID) bioinformatics resources consist of an integrated biological knowledge base and analytical tools aimed at systematically extracting biological meaning from large gene or protein lists (32). DAVID was used to analyze the enriched functions and pathways of the common DEGs at the three time points with $\mathrm{P}<0.05$.

In addition, the expression values of DEGs that participated in the most significant GO functions and KEGG pathways were compared.

\section{Results}

Screening and hierarchical clustering analysis. In order to investigate DEGs in mice with BA at 3,7 and 14 days following viral induction, the publicly available microarray dataset GSE46967 from GEO was used. A total of 306 DEGs were selected in the 3 day samples, 721 DEGs in the 7 day samples and 370 DEGs in the 14 day samples (Fig. 1). From the heat maps (Fig. 2), the results of hierarchical clustering analysis revealed that the case- and control-groups can be separated by the selected DEGs in each group, which means the expression patterns of DEGs screened were significant, and they can be used to distinguish case and control samples. A total of 74 common DEGs were identified between the samples from the different time points (Fig. 3A), and the expression values of all of these common DEGs were upregulated, as compared with in the control samples(Fig. 3B).

Functional annotation analysis of common DEGs. To investigate the alterations in the function of selected common DEGs, DAVID was used to identify the significant GO categories in the biological process. A total of $12 \mathrm{GO}$ terms were observed to be enriched among the 74 DEGs (Table I), of which several significantly enriched functions were associated with immune function, including the defense response, leukocyte migration, cell chemotaxis and leukocyte chemotaxis. C3AR1, CCL3, 


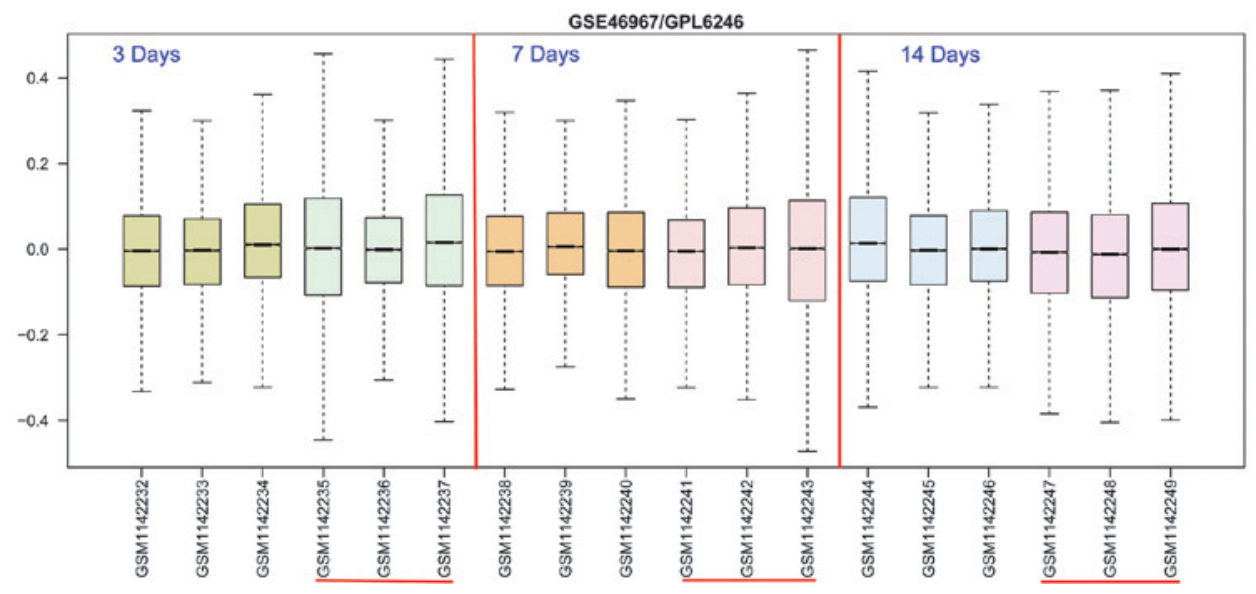

Figure 1. Cassette figure of expression values of DEGs. Case-group is marked by a red horizontal line in each sample.
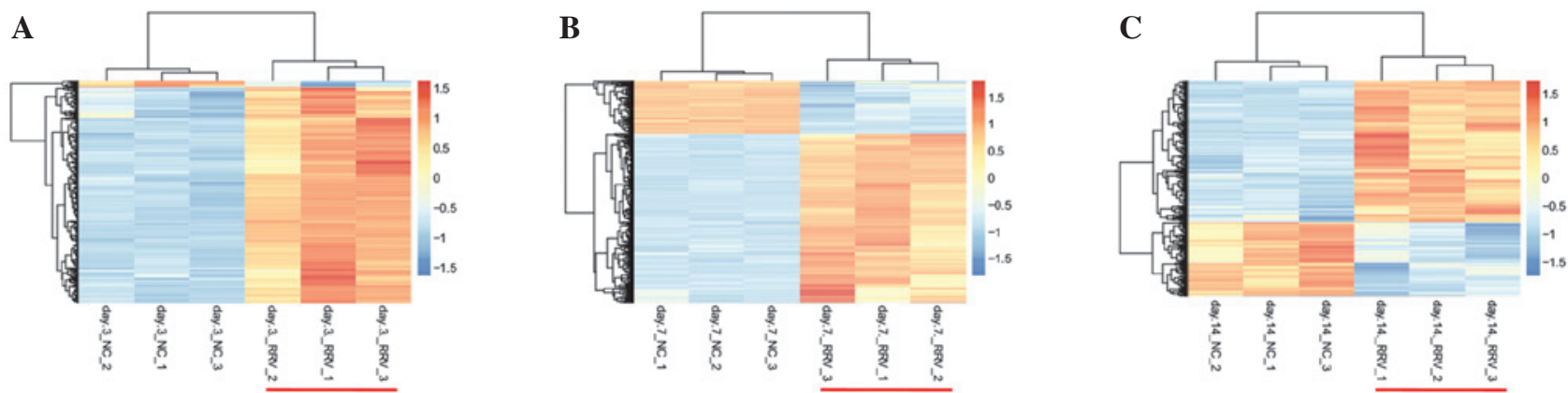

Figure 2. Hierarchical clustering heat maps of the DEGs. Heat maps of DEGs at (A) 3, (B) 7 and (C) 14 days. Color gradient from blue-orange represents expression level (case-group and control-group) from up- to downregulation. Case-group is marked by a red horizontal line in each sample. DEGs, differentially expressed genes.

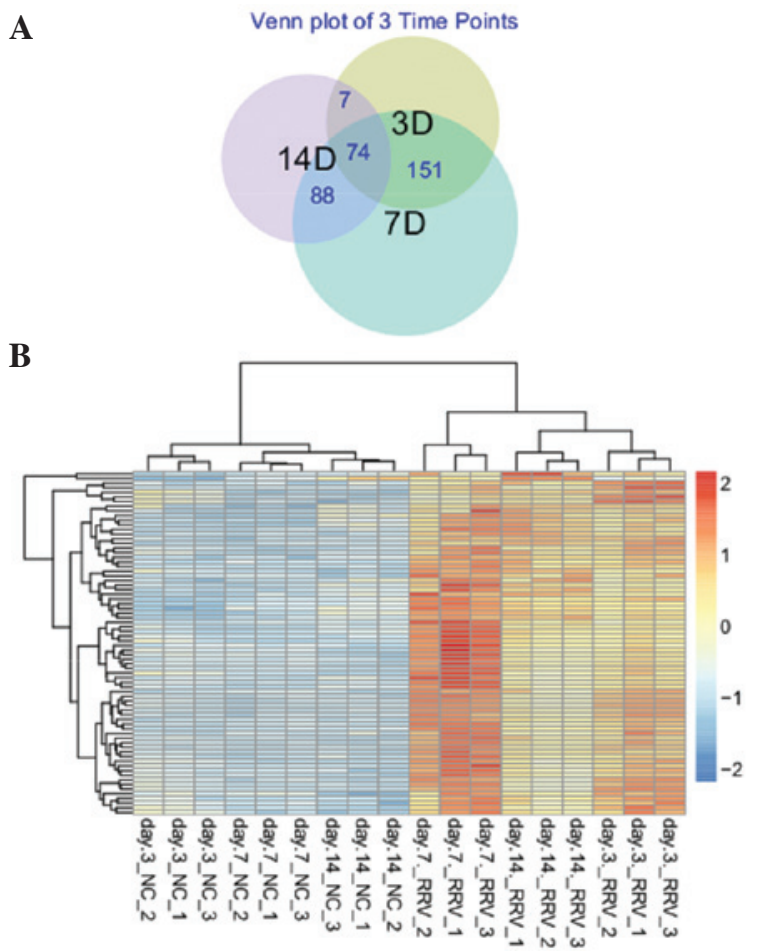

Figure 3. Expression values of the selected 74 common DEGs at three time points. (A) Venn diagram of DEGs from the three time points. (B) Hierarchical clustering heat maps of DEGs. Orange represents upregulation, and blue represents downregulation. DEGs, differentially expressed genes.
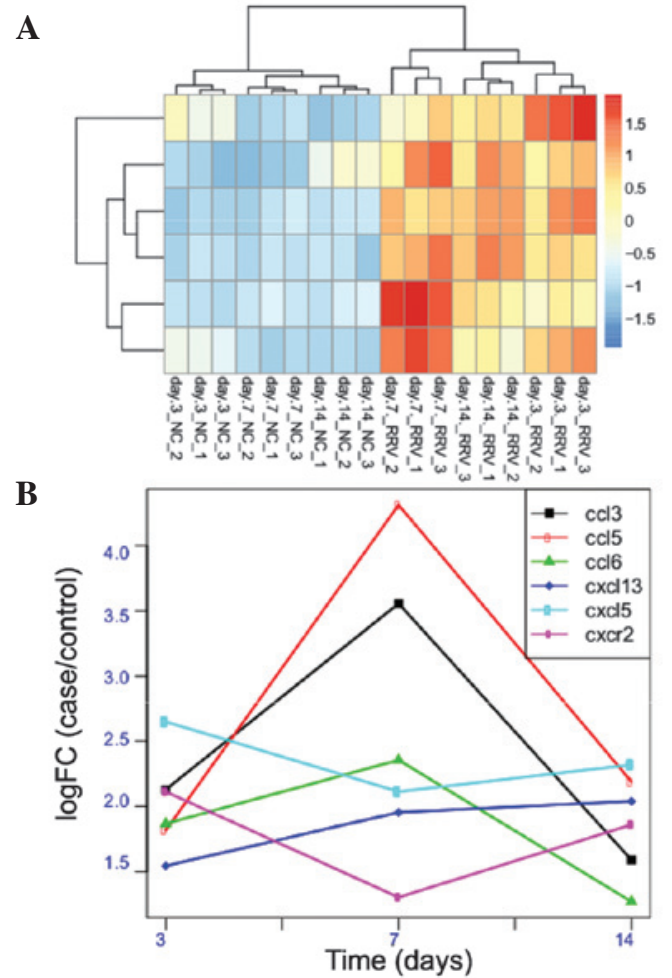

Figure 4. Expression values of the selected 6 DEGs that participate in the significant functions and pathways. (A) Hierarchical clustering heat maps of the 6 DEGs. (B) Expression values of the 6 DEGs at different time points. DEGs, differentially expressed genes. 
Table I. Function enrichment analysis of the common DEGs.

\begin{tabular}{lcc}
\hline Term & Count & FDR \\
\hline GO:0042330 taxis & 13 & $1.67 \times 10^{-13}$ \\
GO:0006935 chemotaxis & 14 & $1.67 \times 10^{-13}$ \\
GO:0006955 immune response & 21 & $1.67 \times 10^{-13}$ \\
GO:0007626 locomotory behavior & 14 & $4.38 \times 10^{-09}$ \\
GO:0006952 defense response & 17 & $5.54 \times 10^{-09}$ \\
GO:0007610 behavior & 14 & $3.07 \times 10^{-06}$ \\
GO:0030595 leukocyte chemotaxis & 6 & $6.90 \times 10^{-05}$ \\
GO:0060326 cell chemotaxis & 6 & $6.90 \times 10^{-05}$ \\
GO:0006954 inflammatory response & 10 & $1.98 \times 10^{-04}$ \\
GO:0030593 neutrophil chemotaxis & 5 & $6.03 \times 10^{-04}$ \\
GO:0009611 response to wounding & 11 & $7.79 \times 10^{-04}$ \\
GO:0050900 leukocyte migration & 6 & $7.86 \times 10^{-04}$ \\
\hline
\end{tabular}

DEGs, differentially expressed genes; GO, gene ontology; FDR, false discovery rate.

C5AR1, CXCL5, FPR1, CXCR2, CCL5, ITGAM, CCL6, CXCL13, CSF3R, IL1B and FCER1G were the genes enriched in the significant functions.

Pathway analysis of common DEGs. To further understand the pathways of the DEGs screened in the current study, DAVID was used to identify the significant pathways. Two pathways (Table II), were identified to be enriched, the 'cytokine-cytokine receptor interaction' and 'chemokine signaling pathway'. The 'cytokine-cytokine receptor interaction' pathway was the most significant pathway with ten genes involved, as follows: IL1R2, CCL3, CXCL5, CXCL13, CCR1, IL1B, CSF3R, CXCR2, CCL5 and CCL6.

Expression values of DEGs participating in significant functions and pathways. A total of 13 DEGs were identified to be associated with the significant functions, and 10 DEGs with the significant pathways. Following the comparison of the two types of DEGs, the following 6 common DEGs were identified: CCL3, CXCL5, CXCL13, CXCR2, CCL5 and CCL6. The expression profile of these 6 DEGs in each of the three samples were analyzed (Fig. 4A). It is observed in the figure that all 6 of the DEGs in the three sample types were significantly overexpressed, and CXCL13 was identified to have a consistent overexpression from the 3 rd day to the 14th day. CCL3, CCL5 and CCL6; however, demonstrated the highest overexpression on the 7th day, but were decreased on the 14th day. CXCL5 and CXCR2 presented the highest overexpression on the $3 \mathrm{rd}$ day (Fig. 4B).

\section{Discussion}

BA is an infantile disorder in which there is obliteration or discontinuity of the extrahepatic biliary tree. If left untreated, it progresses to cirrhosis, liver failure and eventually to mortality, and the current treatment strategies are unsatisfactory $(2,5)$. Previous studies using histological sections of extrahepatic biliary tract tissue from patients, in addition to
Table II. Pathways involved with common DEGs.

\begin{tabular}{lcc}
\hline $\begin{array}{l}\text { Kyoto enrichment of } \\
\text { genes and genomes }\end{array}$ & $\begin{array}{c}\text { Gene } \\
\text { number }\end{array}$ & FDR \\
\hline $\begin{array}{l}\text { mmu04060: } \\
\begin{array}{l}\text { Cytokine-cytokine receptor interaction } \\
\text { mmu04062: }\end{array}\end{array}$ & 10 & 0.003256 \\
\begin{tabular}{l} 
Chemokine signaling pathway \\
\hline
\end{tabular} & 9 & 0.003469 \\
\hline
\end{tabular}

DEGs, differentially expressed genes; FDR, false discovery rate.

experimental studies using the BA mice model, have demonstrated that an inflammatory process appears to contribute to the pathogenesis of BA $(18,33)$. Previous studies that further identify the relevant inflammatory cascade revealed an upregulation in pro-inflammatory cytokines (20). A total of 306 DEGs were identified in the 3 day samples, 721 in the 7 day samples and 370 in the 14 day samples in mice with BA following injection with rhesus rotavirus. In total, 74 common DEGs were selected from the three sample types at different time points, which function predominantly in the immune biological process, including the defense response, leukocyte migration, cell chemotaxis and leukocyte chemotaxis. The 'cytokine-cytokine receptor interaction' and 'chemokine signaling pathway' were identified as the most significantly enriched pathways. A total of six significantly overexpressed genes (CCL3, CXCL5, CXCL13, CXCR2, CCL5 and CCL6) associated with the immune response and cytokine pathways at the three time points were screened, which were investigated in the significant functions and pathways.

Chemokines are small proteins that are vital in immune and inflammatory reactions. Induction of leukocyte migration is the predominant function of the chemokines, which additionally affect angiogenesis, collagen production and the proliferation of hematopoietic precursors (34). CCL3 $(\mathrm{MIP}-1 \alpha)$ is a chemokine produced by activated T-cells, basophilic granulocytes and type $2 \mathrm{~T}$ helper cells (35). The chemokine CCL3 has several functions, including serving a role in cell lysis mediated by natural killer cells, participating in the inflammatory response associated with immunological processes, and mediating the release of the other cytokines (36). Seki et al (37) reported that MIP-1 $\alpha$ had the ability to gather the hepatic stellate and inflammatory cells migrating to the site of injury, so as to function on the liver fibration. Zerfaoui et al (38) demonstrated that CCL3 was able to activate T cells thus producing CCL5 (RANTES). CCL3 and CCL5 have the ability to assemble and to activate eosinophilic granulocytes to release histamine and leukotrienes so as to induce inflammatory cell adherence to the vessel wall (38). Increased levels of CCL5 have been observed in patients with primary biliary cirrhosis, and the therapeutic benefits of fibrates in this biliary disease may be due to the inhibition of this CCL5, with its resultant effects on inflammatory cell migration (39). From the results of the present study, it is suggested that the expression of CCL3 and CCL5 are elevated in a time-dependent manner until 7 days, indicating that these two molecules are critical for the 
pathogenesis of BA in mice. Furthermore, previous studies have demonstrated that the two cytokines are associated with the migration and infiltration of monocytes, memory T lymphocytes and natural killer cells (40-42), so it was speculated that they may be involved in the early recruitment of different subsets of leukocytes in the formation of BA.

The CXCR2 gene was first cloned by Murphy and Tiffany (43) and the chemokine CXCR2, which is produced on the surface of neutrophils and natural killer cells, is also a member of the G-protein superfamily. A previous study demonstrated that CXCR2 may combine with the chemokine CXCL5 in order to activate the downstream effector molecules, and may subsequently mediate signal transduction and cell migration via the allosteric G-protein (44). CXCL5 is a small-molecule type secretory protein, which has a direct chemotaxis for neutrophils and is able to mediate the infiltration of neutrophils, thus promoting the metastasis of liver cancer cells (45). Li et al (46) reported that CXCL5 contributed to the formation of tumor vessels by activating certain signaling pathways, such as the protein kinase B, signal transduction and activating transcription factor pathways (47). In the present study, the variation tendency of the CXCL5 expression level was similar with that of CXCR2 and their expression peaked at 3 days. This phenomenon may indicate that the chemokine receptor CXCR2 and CXCL5 may combine, in order to affect inflammation in mice with BA by collecting leukocytes.

CXCL13 is a chemokine produced by B-cells and is critical for targets seeking B-cell and B-cell zone formation in lymphatic tissue (48). A previous study indicated that modulation of the inflammatory signaling cascade, particularly cytokine secretion, may attenuate the severity of BA in the murine model. Shivakumar et al demonstrated that RRV-infected, interferon $\gamma$-deficient Bulb/c mice had a lower mortality rate than wild type animals (48). Anesl et al (49) demonstrated that the natural antibody production in mice deficient in CXCL13 was markedly reduced, and no response was triggered to a bacterial antigen by intraperitoneal injection in this case. In addition, the roles of CXCL13 have been observed in other immune system-associated diseases. Hjelmstrom et al (50) observed that CXCL13 was overexpressed in transgenic mice suffering from chronic inflammation induced by lymphotoxin- $\alpha$ (50). Based on the data from the current study, the expression levels of CXCL13 were increased until 14 days in mice with BA. Elevated levels of CXCL13 is not unique to active BA, but it may serve as a marker of disease activity or response to treatment in combination with other diagnostic markers.

Notably, low expression levels of CCL6 were observed in BA-positive mice. It was hypothesized that the chemokine CCL6 may serve an important function in the progression of BA, however further study is required in order to further elucidate the mechanisms.

In conclusion, CCL3, CCL5, CXCL5, CXCR2, CXCL13 and CCL6, are suggested to be involved in the pathogenesis of BA, but serve different roles. The current study provided a basis for future experiments with the potential use of RRV-injected mice, which may enhance understanding of the pathogenesis of BA and lead to the development of novel therapeutic strategies.

\section{References}

1. Mack CL, Feldman AG and Sokol RJ: Clues to the etiology of bile duct injury in biliary atresia. Semin Liver Dis 32: 307-316, 2012.

2. Petersen C: Pathogenesis and treatment opportunities for biliary atresia. Clin Liver Dis 10: 73-88, 2006.

3. Jimenez-Rivera C, Jolin-Dahel KS, Fortinsky KJ, Gozdyra P and Benchimol EI: International incidence and outcomes of biliary atresia. J Pediatr Gastroenterol Nutr 56: 344-354, 2013.

4. Davenport M, Ong E, Sharif K, et al: Biliary atresia in England and Wales: results of centralization and new benchmark. J Pediatr Surg 46: 1689-1694, 2011.

5. Mendoza MM, Chiang JH, Lee SY, et al: Reappraise the effect of redo-Kasai for recurrent jaundice following Kasai operation for biliary atresia in the era of liver transplantation. Pediatr Surg Int 28: 861-864, 2012.

6. Bijl EJ, Bharwani KD, Houwen RH and de Man RA: The long-term outcome of the Kasai operation in patients with biliary atresia: a systematic review. Neth J Med 71: 170-173, 2013.

7. Cronin DC, Squires J, Squires R, Mazariegos G and Lantos JD: Parental refusal of a liver transplant for a child with biliary atresia. Pediatrics 131: 141-146, 2013.

8. Shimadera S, Iwai N, Deguchi E, Kimura O, Fumino S and Yokoyama T: The inv mouse as an experimental model of biliary atresia. J Pediatr Surg 42: 1555-1560, 2007.

9. Mack CL, Falta MT, Sullivan AK, et al: Oligoclonal expansions of $\mathrm{CD}^{+}$and $\mathrm{CD}^{+}$T-cells in the target organ of patients with biliary atresia. Gastroenterology 133: 278-287, 2007.

10. de Carvalho E, Ivantes CA and Bezerra JA: Extrahepatic biliary atresia: current concepts and future directions. J Pediatr (Rio J) 83: 105-120, 2007.

11. Soomro GB, Abbas Z, Hassan M, Luck N, Memon Y and Khan AW: Is there any association of extra hepatic biliary atresia with cytomegalovirus or other infections. J Pak Med Assoc 61: 281-283, 2011.

12. Mahjoub F, Shahsiah R, Ardalan FA, et al: Detection of Epstein Barr virus by chromogenic in situ hybridization in cases of extra-hepatic biliary atresia. Diagn Pathol 3: 19, 2008.

13. Schaffer K, Hassan J, Staines A, et al: Surveillance of Epstein-Barr virus loads in adult liver transplantation: associations with age, sex, posttransplant times, and transplant indications. Liver Transpl 17: 1420-1426, 2011.

14. Whitington PF, Malladi P, Melin-Aldana H, Azzam R, Mack CL and Sahai A: Expression of osteopontin correlates with portal biliary proliferation and fibrosis in biliary atresia. Pediatr Res 57: 837-844, 2005.

15. Mack CL. The pathogenesis of biliary atresia: evidence for a virus-induced autoimmune disease. Semin Liver Dis 27: 233-242, 2007.

16. Leonhardt J, Stanulla M, Von Wasielewski R, et al: Gene expression profile of the infective murine model for biliary atresia. Pediatr Surg Int 22: 84-89, 2006.

17. Schreiber RA and Kleinman RE: Biliary atresia. J Pediatr Gastroenterol Nutr 35 (Suppl 1): S11-S16, 2002.

18. Mack CL, Tucker RM, Sokol RJ, et al: Biliary atresia is associated with $\mathrm{CD} 4^{+} \mathrm{Th} 1$ cell-mediated portal tract inflammation. Pediatr Res 56: 79-87, 2004.

19. Lages CS, Simmons J, Chougnet CA and Miethke AG: Regulatory T cells control the CD8 adaptive immune response at the time of ductal obstruction in experimental biliary atresia. Hepatology 56: 219-227, 2012.

20. Bezerra JA, Tiao G, Ryckman FC, et al: Genetic induction of proinflammatory immunity in children with biliary atresia. Lancet 360: 1653-1659, 2002.

21. Nakamura K and Tanoue A: Etiology of biliary atresia as a developmental anomaly: recent advances. J Hepatobiliary Pancreat Sci 20: 459-464, 2013.

22. Hirschfield GM, Liu X, Xu C, et al: Primary biliary cirrhosis associated with HLA, IL12A, and IL12RB2 variants. N Engl J Med 360: 2544-2555, 2009.

23. Barrett T, Troup DB, Wilhite SE, et al: NCBI GEO: mining tens of millions of expression profiles - database and tools update. Nucleic Acids Res 35: D760-D765, 2007.

24. Fujita A, Sato JR, Rodrigues Lde O, Ferreira CE and Sogayar MC Evaluating different methods of microarray data normalization. BMC bioinformatics 7: 469, 2006.

25. Anders S, McCarthy DJ, Chen Y, et al: Count-based differential expression analysis of RNA sequencing data using $\mathrm{R}$ and Bioconductor. Nat Protoc 8: 1765-1786, 2013. 
26. Benjamini $\mathrm{Y}$ and Hochberg Y: Controlling the false discovery rate: a practical and powerful approach to multiple testing. J R Stat Soc Series B Stat Methodol 57: 289-300, 1995.

27. Langfelder $P$ and Horvath S: Fast R functions for robust correlations and hierarchical clustering. J Stat Softw 46: 11, 2012.

28. Mukherjee S, Chen Z and Gangopadhyay A: A privacy-preserving technique for Euclidean distance-based mining algorithms using Fourier-related transforms. VLDB J 15: 293-315, 2006.

29. Pirooznia M, Nagarajan V and Deng Y: GeneVenn - A web application for comparing gene lists using Venn diagrams. Bioinformation 1: 420-422, 2007.

30. Hulsegge I, Kommadath A and Smits MA. Globaltest and GOEAST: two different approaches for Gene Ontology analysis. BMC Proc 3 (Suppl 4): S10, 2009.

31. Kanehisa $M$ and Goto S: KEGG: kyoto encyclopedia of genes and genomes. Nucleic Acids Res 28: 27-30, 2000.

32. Huang da W, Sherman BT and Lempicki RA: Systematic and integrative analysis of large gene lists using DAVID bioinformatics resources. Nat Protoc 4: 44-57, 2008.

33. Mack CL, Tucker RM, Sokol RJ and Kotzin BL: Armed CD4 Th1 effector cells and activated macrophages participate in bile duct injury in murine biliary atresia. Clin Immunol 115: 200-209, 2005.

34. Carmeliet P: Angiogenesis in health and disease. Nat Med 9: 653-660, 2003.

35. Ramos CDL, Canetti C, Souto JT, et al: MIP-alpha[CCL3] acting on the CCR1 receptor mediates neutrophil migration in immune inflammation via sequential release of TNF-alpha and LTB4. J Leukoc Biol 78: 167-177, 2005.

36. Ekman AK, Fransson M, Rydberg C, Adner M and Cardell LO: Nasal challenge with LPS stimulates the release of macrophage inflammatory protein 1alpha. Int Arch Allergy Immunol 149: 154-160, 2009.

37. Seki E, De Minicis S, Gwak GY, et al: CCR1 and CCR5 promote hepatic fibrosis in mice. J Clin Invest 119: 1858-1870, 2009

38. Zerfaoui M, Naura AS, Errami Y, et al: Effects of PARP-1 deficiency on airway inflammatory cell recruitment in response to LPS or TNF: differential effects on CXCR2 ligands and Duffy antigen receptor for chemokines. J Leukoc Biol 86: 1385-1392, 2009 .
39. Hirano Y, Hirano F, Fujii H and Makino I: Fibrates suppress chenodeoxycholic acid-induced RANTES expression through inhibition of NF-kappaB activation. Eur J Pharmacol 448: 19-26, 2002.

40. Yamamoto S, Shimizu S, Kiyonaka S, et al: TRPM2-mediated $\mathrm{Ca}^{2+}$ influx induces chemokine production in monocytes that aggravates inflammatory neutrophil infiltration. Nat Med 14: 738-747, 2008

41. Schlecker E, Stojanovic A, Eisen C, et al: Tumor-infiltrating monocytic myeloid-derived suppressor cells mediate CCR5-dependent recruitment of regulatory $\mathrm{T}$ cells favoring tumor growth. J Immunol 189: 5602-5611, 2012.

42. Robertson MJ: Role of chemokines in the biology of natural killer cells. J Leukoc Biol 71: 173-183, 2002.

43. Murphy PM and Tiffany HL: Cloning of complementary DNA encoding a functional human interleukin- 8 receptor. Science 253 : 1280-1283, 1991.

44. Stillie R, Faroog SM, Gordon JR and Stadnyk AW: The functional significance behind expressing two IL-8 receptor types on PMN. J Leukoc Biol 86: 529-543, 2009.

45. Okabe H, Beppu T, Ueda M, et al: Identification of CXCL5/ENA-78 as a factor involved in the interaction between cholangiocarcinoma cells and cancer-associated fibroblasts. Int J Cancer 131: 2234-2241, 2012

46. Li A, King J, Moro A, et al: Overexpression of CXCL5 is associated with poor survival in patients with pancreatic cancer. Am J Pathol 178: 1340-1349, 2011.

47. van de Pavert SA, Olivier BJ, Goverse G, et al: Chemokine CXCL13 is essential for lymph node initiation and is induced by retinoic acid and neuronal stimulation. Nat Immunol 10: 1193-1199, 2009.

48. Shivakumar P, Campbell KM, Sabla GE, et al: Obstruction of extrahepatic bile ducts by lymphocytes is regulated by IFN- $\gamma$ in experimental biliary atresia. J Clin Invest 114: 322-329, 2004

49. Ansel KM, Harris RB and Cyster JG: CXCL13 is required for B1 cell homing, natural antibody production, and body cavity immunity. Immunity 16: 67-76, 2002.

50. Hjelmström P, Fjell J, Nakagawa T, Sacca R, Cuff CA and Ruddle NH: Lymphoid tissue homing chemokines are expressed in chronic inflammation. Am J Pathol 156: 1133-1138, 2000. 